\title{
NUMERICAL MODELING OF OPTICAL PROPERTIES INSIDE THE SURFZONE
}

\author{
Fengyan Shi ${ }^{1}$, Gangfeng $\mathrm{Ma}^{1}$, James T. Kirby ${ }^{1}$
}

\begin{abstract}
This paper provides a review of our recent developments in numerical models for predicting physical processes related to optical properties inside the surfzone. Model components in the developments include the bubble entrainment model, 2D and 3D multiphase two-fluid models for modeling quiescent phase of bubbles, turbulence models with influences of bubbles, bubble coalescence and breakup models used in the two-fluid models, and foam model for predicting foam patch generation and evolution inside the surfzone. The paper summarizes theories used in the model components and shows some numerical results from model tests.

Keywords: air bubble; foam; multiphase model; Navier-Stakes solver; surfzone
\end{abstract}

\section{INTRODUCTION}

Wave breaking inside the surfzone entrains large volumes of air bubbles into the water column. This air volume subsequently evolves into a distribution of bubble sizes which interact with the fluid turbulence and are advected by the organized flow. Bubbles rising to the surface create a foam layer on the water surface. The optical penetrability is affected by irradiance attenuation and scattering due to air bubbles and sediment particles, and path occlusion by the presence of foam on the water surface.

Early investigations on bubble entrainment and evolution under surfzone breaking waves are mostly through laboratory measurements (e.g., Thorpe, 1982; Lamarre and Melville, 1991, 1994; Garrett et al., 2000; Terrill et al., 2001; Deane and Stokes, 1999, 2002; Hoque, 2002; Mori et al., 2007). The photographic studies on air entrainment mechanism by Deane and Stokes (2002) suggested that the lifetime of wave-generated bubbles can be categorized into two phases. The first phase is called the acoustic phase, during which bubbles are entrained and fragmented inside the breaking wave crest. The second phase happens after bubble creation processes cease and the newly formed bubbles evolve under the influence of turbulent diffusion, advection, buoyant degassing, and dissolution. Because this phase is acoustically quiescent, it is called the quiescent phase. The analysis on bubble size distribution under laboratory plunging waves revealed that the bubble creation is driven by two large-scale processes: the jet/wave-face interaction and the collapsing cavity. The first process is primarily responsible for the formation of small bubbles with radius less than Hinze scale $(\sim 1 \mathrm{~mm})$, while the latter is mainly responsible for the generation of bubbles larger than Hinze scale. The bubble size spectrum of their measurements satisfies a $-3 / 2$ power law for small bubbles and a $-10 / 3$ power law for large bubbles.

Compared with laboratory experiments, numerical studies of wave breaking induced two-phase bubbly flow field are rare probably because of the lack of

${ }^{1}$ Center for Applied Coastal Research, University of Delaware, Newark, DE 19716, USA 
robust and comprehensive bubble entrainment models. The recent studies on two-phase Navier-Stokes solvers (e.g., Liu and Lin, 2008) showed the possibilities for directly predicting air packet entrainment and bubble evolution. The studies also indicated some difficulties in modeling air packet breakup, and small bubble entrainment and evolution processes because the requirement for higher resolution in both time and space makes a model computationally unaffordable. In contrast with models that resolve individual bubbles, models based on volume averaged properties associated with bubble population are efficient. Carrica et al. (1999) developed a polydisperse two-fluid model to study the bubbly flow field around a surface ship, but without taking into account bubble entrainment processes. The bubbles were introduced into the computation through measured data in plunging jet experiments. Moraga et al. (2008) presented a sub-grid model that detects the location of the air bubble entrainment region. The localized region of high void fraction is bounded by the surface at which the downward liquid velocity reaches a certain value $(0.22 \mathrm{~m} / \mathrm{s}$ was used in Moraga et al.'s application). The initial bubble size distribution in the localized region follows the bubble size spectrum measured by Deane and Stokes (2002) who suggested that, at the beginning of the quiescent phase, the size spectrum follows a certain power-law scaling with bubble radius.

Numerical studies of foam dynamics are mostly restricted to modeling of foam microstructures and their evolutions which are determined by the interfacial forces occurring at the film level (Davini, 2010). Practical applications of such models in larger scales (surfzone scale) are rarely found in literature. The formation, growing, drainage, bursting and transport of foams involve complex physical processes and are related to chemical properties (surfactant) of fluid as well. From the viewpoint of predicting foam patches at the surfzone scale, the crucial problem may be how to estimate the source/sink of foam in the surfzone environment. A model for a practical use in predicting foam coverage and transport inside the surfzone may start with a simple process-based transport model with incorporation of existing empirical formulations, parameterization of results from a bubble prediction model into calculations of source/sink terms in the model.

The aim of our study is to develop a tested model of optical properties in the surfzone to assess how optical properties are related to individual breaking wave crests and wave-driven surfzone circulation. We have been developing and testing several model components associated predictions of optical properties, including multiphase bubble model, suspended sediment transport model, foam layer model and surfzone irradiance model. In the present paper, we will focus on introducing the recent progresses in developing of multiphase air bubble models and a foam model.

\section{BUBBLE ENTRAINMENT MODELS}

Shi et al. $(2008,2010)$ used a simple source function to model bubbles entrained by breaking waves. The initial bubble entrainment is prescribed by connecting the production of turbulent kinetic energy at the air-water interface 
and the bubble number intensity with certain bubble size spectra observed by Deane and Stokes (2002). The increment of initial bubble number per unit radius increment can be written as

$$
d N_{b, i}=a_{b} P_{r} D_{i} d t, \quad P_{r}>P_{r 0}
$$

where $P_{r}$ is the shear production term, i.e., $P_{r}=\mu_{t}|\mathbf{S}|^{2}$, in which $\mathbf{S}$ is the rate of strain and $\mu_{t}$ is the eddy viscosity, $P_{r 0}$ is a threshold for the onset air entrainment, $D_{i}$ is the bubble size probability function. Based on Deane and Stokes (2002), the bubble density per unit radius increment can by calculated by

$$
N= \begin{cases}N_{H}\left(\frac{r_{b}}{r_{H}}\right)^{-3 / 2}, & r_{b, \min } \leq r_{b} \leq r_{H} \\ N_{H}\left(\frac{r_{b}}{r_{H}}\right)^{-10 / 3}, & r_{H}<r_{b} \leq r_{b, \max }\end{cases}
$$

where $r_{b, \min }$ and $r_{b, \max }$ represent respectively the minimum and maximum bubble radius considered, $N_{H}$ is the bubble density per unit radius increment at the Hinze scale $r_{H}$ (Hinze, 1955).

The air entrainment parameter calibrated using the void fraction measured in laboratory experiments predicted the evolution of void fraction in the water column with reasonable agreements between model results and the measured data in Lamarre and Melville (1991). However, the theoretically unjustified air entrainment formulation with the dimensional parameter $a_{b}$ suggest some uncertainties in use of this model for general scales of bubble entrainment applications.

Recently, Ma et al. (2010) proposed an air entrainment model according to the physical analysis of bubble creation processes in a wave breaking environment. The air entrainment formulation was based on the assumption that bubble creation under breaking waves is determined by the turbulent dissipation rate $\epsilon$ with a linear relationship between the energy required for bubble creation and the dissipation rate:

$$
E_{b}\left(r_{b}\right) B\left(r_{b}\right)=c_{b} \rho_{l} \epsilon
$$

where $E_{b}$ is the energy repuired to entrain a single bubble with a radius of $r_{b}, B\left(r_{b}\right)$ is the rate of bubble creation per cubic meter, $c_{b}$ is air entrainment coefficient, $\rho_{l}$ is the density of liquid. For a given entrained air volume, integrating (3) over all sizes of bubbles with the bubble size spectrum given by Deane and Stokes (2002), the bubble entrainment rate for the bubble group $i$ can be expressed by

$$
B\left(a_{i}\right)=\frac{c_{b}}{4 \pi}\left(\frac{\sigma}{\rho_{l}}\right)^{-1} \frac{S\left(r_{b i}\right) \Delta r_{b i}}{\sum_{i=1}^{N G} r_{b i}^{2} S\left(a_{i}\right) \Delta r_{b i}} \epsilon
$$

where $S\left(r_{b}\right)$ is the bubble size spectrum, $\sigma$ is the surface tension, $N G$ is the number of bubble groups. The parameter $c_{b}$ is dimensionless and can be calibrated using data measured in field or laboratory experiments. 


\section{MULTIPHASE TWO-FLUID MODELS FOR MODELING QUIESCENT PHASE OF AIR BUBBLES}

Two approaches have been developed for modeling quiescent phase of air bubbles in the study. One is a 2DV quasi-multiphase model based on Buscaglia et al. (2002) and implemented in the 2D VOF model RIPPLE (Kothe et al., 1991). The other is a 3D multiphase model based on Carrica et al. (1998) using the 3D VOF code TRUCHAS (Francois et al., 2006) as the basic framework.

The governing equations in the 2DV model (Shi et al., 2008, 2010) include mass conservation and momentum equations for the mixture fluid phase:

$$
\begin{gathered}
\nabla \cdot \mathbf{u}_{m}=0 \\
\frac{\partial \mathbf{u}_{m}}{\partial t}+\mathbf{u}_{m} \cdot \nabla \mathbf{u}_{m}+\frac{1}{\rho_{0}} \nabla P_{m}=\frac{1}{\rho_{0}} \nabla \cdot\left(2 \mu_{t} \mathbf{S}\right)-\frac{\rho_{m}}{\rho_{0}} g \mathbf{k}
\end{gathered}
$$

where $\mathbf{u}_{m}, P_{m}$ and $\rho_{m}$ represent the mixture quantities of fluid velocity, pressure and density, respectively, $\mathbf{k}$ is a vertical unit vector, $\rho_{0}$ is the so called reference density which has replaced $\rho_{m}$ in all terms but the gravity term using the Boussinesq approximation.

The influence of air bubble on the mixture fluid phase is reflected by the last term in (6), the buoyancy force, which can be evaluated by

$$
\frac{\rho_{m}}{\rho_{0}} g \mathbf{k}=\left(1-\alpha_{b}\right) g \mathbf{k}
$$

where $\alpha_{b}$ is the volume fraction of bubbles following the definition in Drew and Passman (1998).

The equations for the bubble phase include the equations of the gas molar concentration and bubble number intensity with different bubble sizes. Mass bin $i$ of the bubble population is calculated using simple advection-diffusion equations given by

$$
\begin{gathered}
\frac{\partial C_{b, i}}{\partial t}+\nabla \cdot\left(C_{b, i} \mathbf{u}_{g}\right)=\mathcal{E}_{c, i}+\mathcal{S}_{c, i}+\nabla \cdot\left(\mathcal{D}_{g} \nabla C_{b, i}\right) \\
\frac{\partial N_{b, i}}{\partial t}+\nabla \cdot\left(N_{b, i} \mathbf{u}_{g}\right)=\mathcal{E}_{n, i}+\mathcal{S}_{n, i}+\nabla \cdot\left(\mathcal{D}_{g} \nabla N_{b, i}\right)
\end{gathered}
$$

where $C_{b, i}$ and $N_{b, i}$ represent, respectively, the gas molar concentration and bubble number per unit volume for bubble size $i, \mathbf{u}_{\mathrm{g}}$ is the bubble advection velocity which is the combination of the mixture fluid velocity and the bubble-slip velocity evaluated using Clift et al. (1978). $\mathcal{E}_{c, i}$ and $\mathcal{E}_{n, i}$ are source terms associated with bubble entrainment. $\mathcal{S}_{c, i}$ and $\mathcal{S}_{n, i}$ are source/sink terms associated with inter-group adjustment of bubble quantity between different component $i$ caused by bubble size changes due to pressure change, bubble breakup and coalescence. $\mathcal{D}_{g}$ is the dispersion coefficient associated with the turbulence and bubble-bubble interaction. In the connection between $C_{b}$ and $N_{b}$, the ideal gas law is used. 
In the 3D two-fluid model, Carrica et al.'s (1999) approach for simulating bubble plumes was adopted. The model is based on the multiphase theory with neglecting the inertia and shear stress tensors for the gas phase due to the relatively small gas volume and density. The governing equations include the continuity equation for the liquid phase:

$$
\frac{\partial\left(\alpha_{l} \rho_{l}\right)}{\partial t}+\nabla \cdot\left(\alpha_{l} \rho_{l} \mathbf{u}_{l}\right)=0
$$

the momentum equation for the liquid phase:

$$
\begin{aligned}
& \frac{\partial\left(\alpha_{l} \rho_{l} \mathbf{u}_{l}\right)}{\partial t}+\nabla \cdot\left(\alpha_{l} \rho_{l} \mathbf{u}_{l} \mathbf{u}_{l}\right)=-\alpha_{l} \nabla p+\alpha_{l} \rho_{l} \mathbf{g} \\
& +\nabla \cdot\left[\alpha_{l} \mu_{e f f, l}\left(\nabla \mathbf{u}_{l}+\nabla^{T} \mathbf{u}_{l}\right)\right]+\mathbf{M}_{g l}
\end{aligned}
$$

the continuity equation of bubble number density $N_{g, i}$ for bubble group $i$ :

$$
\frac{\partial N_{g, i}}{\partial t}+\nabla \cdot\left(\mathbf{u}_{g, i} N_{g, i}\right)=\mathcal{E}_{n, i}+\mathcal{S}_{n, i}
$$

and the momentum equation of bubbles for bubble group $i$ :

$$
-\alpha_{g, i} \nabla p+\alpha_{g, i} \rho_{g, i} \mathbf{g}+\mathbf{M}_{l g, i}=0
$$

where $\rho_{l}$ is liquid density, $\mathbf{u}_{l}$ is liquid velocity, $p$ is pressure which is identical in phases, $\rho_{g, i}$ is the bubble density of group $i, \mathbf{g}$ is gravity, $\mu_{e f f, l}$ is the effective viscosity of liquid phase, $\mathbf{u}_{g, i}$ is bubble velocity, $\mathbf{M}_{g l}$ and $\mathbf{M}_{l g, i}$ are the momentum transfer between phases, including effects of virtual mass, lift force and drag force (Clift et al., 1978).

\section{TURBULENCE MODEL WITH INFLUENCES OF BUBBLES}

The nonlinear $k-\epsilon$ turbulence model and the LES model were used, respectively, in the 2DV and 3D two-fluid models. In Ma et al. (2010), the $k-\epsilon$ model was also implemented as an option for 2D applications.

The nonlinear $k-\epsilon$ model can be expressed in a general form for applications of two-phase bubbly flows. The conservation equations of turbulent kinetic energy $k$ and turbulence dissipation rate $\epsilon$ are formulated as

$$
\begin{gathered}
\frac{\partial\left(\alpha_{l} \rho_{l} k\right)}{\partial t}+\nabla \cdot\left(\alpha_{l} \rho_{l} \mathbf{u}_{l} k\right)=\nabla \cdot\left(\alpha_{l} \frac{\mu_{T, l}}{\sigma_{k}} \nabla k\right)+\alpha_{l}\left(G-\rho_{l} \epsilon\right)+S_{b k} \\
\frac{\partial\left(\alpha_{l} \rho_{l} \epsilon\right)}{\partial t}+\nabla \cdot\left(\alpha_{l} \rho_{l} \mathbf{u}_{l} \epsilon\right)=\nabla \cdot\left(\alpha_{l} \frac{\mu_{T, l}}{\sigma_{\epsilon}} \nabla \epsilon\right)+\alpha_{l}\left(C_{\epsilon 1} \frac{\epsilon}{k} G-C_{\epsilon 2} \rho_{l} \frac{\epsilon^{2}}{k}\right)+S_{b \epsilon}
\end{gathered}
$$

where the standard constants for $k-\epsilon$ model are $\sigma_{k}=1.0, \sigma_{\epsilon}=1.3, C_{\epsilon 1}=$ $1.44, C_{\epsilon 2}=1.92$. In the 2D applications of Ma et al. (2010), the term $G$ is the production of turbulent kinetic energy and described by $G=\tau_{l}: \nabla \mathbf{u}_{l}$ in which 
$\tau_{l}$ is shear stress of liquid phase which is calculated from the nonlinear Reynolds stress (Lin and Liu, 1998). In the 2DV model of Shi et al. (2010), the liquid phase in (14) and (15) is represented by the mixture phase. Therefore, $\rho_{l}$ is replaced by $\rho_{m}$, and $G=0$ and $\alpha_{l}=1$. The last two terms $S_{b k}$ and $S_{b \epsilon}$ are bubble induced turbulence production. They were evaluated using approaches of Troshko and Hassan (2001) in Ma et al. (2010) and Kataoka and Serizawa (1989) in Shi et al. (2010).

In the LES model implemented in the 3D two-fluid model (Ma et al., 2010), the effective viscosity of the liquid phase $\mu_{e f f, l}$ is composed of three contributions: the molecular viscosity $\mu_{L, l}$, the turbulent viscosity $\mu_{T, l}$ and an extra term due to bubble induced turbulence $\mu_{B I T, l}$ (Deen, 2001).

$$
\mu_{e f f, l}=\mu_{L, l}+\mu_{T, l}+\mu_{B I T, l}
$$

The turbulent eddy viscosity is calculated by the Smagorinsky (1963) sub-grid model.

$$
\mu_{T, l}=\rho_{l}\left(C_{s} \Delta\right)^{2}|\mathbf{S}|
$$

where $C_{s}$ is a model constant with a value of 0.1 and $\Delta$ is the filter width.

The bubble induced turbulent viscosity is calculated by the model proposed by Sato and Sekoguchi (1975)

$$
\mu_{B I T, l}=\rho_{l} C_{\mu, B I T} \sum_{i=1}^{N G} \alpha_{g, i} d_{b i}\left|\mathbf{u}_{r}\right|
$$

where the model constant $C_{\mu, B I T}$ is equal to 0.6.

\section{BUBBLE COALESCENCE AND BREAKUP MODELS}

The intergroup mass transfer accounts for bubble coalescence and breakup can be expressed by

$$
\mathcal{S}_{n, i}=\chi_{i}^{+}-\chi_{i}^{-}+\beta_{i}^{+}-\beta_{i}^{-}
$$

where $\chi_{i}^{ \pm}$and $\beta_{i}^{ \pm}$represent source/sink due to the coalescence and breakup, respectively.

In Shi et al. (2010), the intergroup mass transfer due to bubble coalescence was evaluated using the algorithm of Prince and Blanch (1990). Bubble coalescence was not taken into account in the 3D model of Ma et al. (2010) because the coalescence effect was found to be insignificant in model applications. The bubble breakup model used in Shi et al. (2010) is the model of Luo and Svendsen (1996) who assumed that the breakup splits a bubble into two identical daughter bubbles. Ma et al. (2010) implemented the approach of Martínez-Bazán et al. (1999). The discussions on different formulations of bubble coalescence and breakup may be found in Lasheras et al. (2002) and Chen et al. (2005). 


\section{FOAM MODEL}

The foam layer model is governed by the mass and momentum equations associated with foam thickness $\xi$ and foam transport velocity $\mathbf{U}_{\text {foam: }}$ :

$$
\frac{\partial \xi}{\partial t}+\nabla \cdot\left(\xi \mathbf{U}_{\text {foam }}\right)=S_{\text {gen }}+S_{\text {bur }},
$$

and

$$
\boldsymbol{\tau}^{s}\left(\mathbf{U}_{\text {foam }}, \mathbf{U}_{\text {flow }}\right)+\boldsymbol{\tau}^{b}\left(\mathbf{U}_{\text {foam }}, \mathbf{U}_{\text {wind }}\right)=0
$$

where $S_{\text {gen }}$ and $S_{\text {bur }}$ are source terms for foam generation and foam bursting, respectively. $S_{\text {gen }}$ can be evaluated using the air volume from degassing of water column, which can be parameterized by the two-fluid model. $S_{\text {bur }}$ can be calculated using an empirical formula such as in Weaire and Hutzler (1999). $\tau^{s}$ and $\tau^{b}$ are, respectively, drag forces at the foam surface and at the foam-wave interface. They are functions of wind velocity $\mathbf{U}_{\text {wind }}$ and flow velocity $\mathbf{U}_{\text {flow }}$. The quadratic stress law may be used to estimate $\tau^{s}$ and $\tau^{b}$. The foam layer model may be coupled with a Boussinesq wave model.

\section{APPLICATIONS}

The capabilities of the models developed in Shi et al. (2010) and Ma et al. (2010) have been tested by comparing to experimental data in laboratory conditions. Shi et al. (2010) showed a simulation of air bubbles measured in the breaking wave experiment by Lamarre and Melville (1991). Moments of the void fraction field defined in Lamarre and Melville (1991) were calculated based on numerical results of void fraction and compared to measurements. Figure 1 shows model/data comparisons of three moments: (a) the normalized total cross-sectional area $A$ of the bubble plume above the void fraction threshold $0.3 \%$ (b) the normalized volume of air entrained per unit width, $V$ and (c) the averaged void fraction, $\bar{\alpha}_{b}$. The model predicted a parabolic-like evolution of the void fraction area $A$, which has a similar trend as shown by the data fitted curve. The area $A$ was over-predicted at the beginning of wave breaking, and a more moderate increase in $A$ can be found in the early time in the wave period, compared with the data fitted line. The comparison of the normalized air volume shown in Figure 1 (b) indicates an underprediction of air entrainment at the beginning of breaking, which is consistent with the absence of a large entrained pocket of air in the numerical simulation. An underprediction of the average void fraction can also be found at the beginning of wave breaking as shown in Figure 1 (c) In general, the model predictions of the magnitude and evolutionary trend of the average void fraction are in reasonable agreement with the data. The underprediction at the beginning of wave breaking was expected because the model does not account for large air pockets in the continuum phase.

Ma et al. (2010) carried out a 3D modeling of an oscillatory bubble plume measured in an experimental vessel by Becker et al. (1994). The numerical results indicated that the 3D model predicted well the flow pattern, bubble plume-induced 

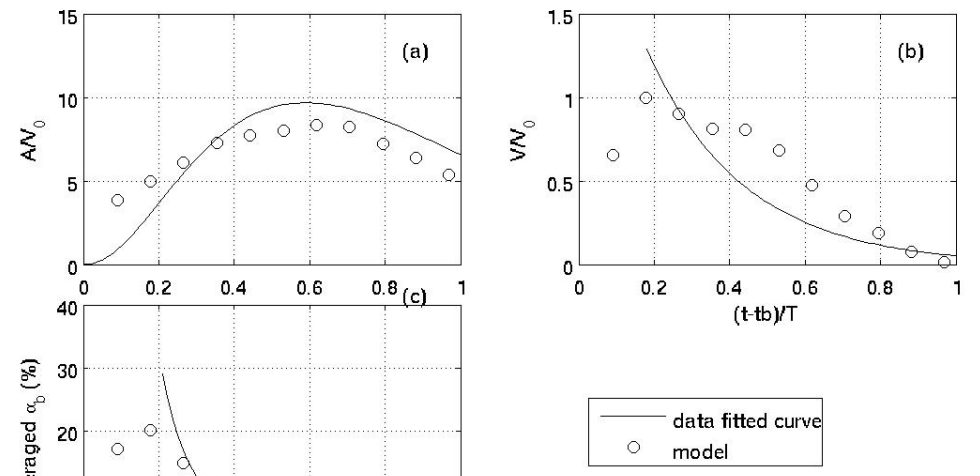

Figure 1. Moments calculated using the void fraction threshold of $0.3 \%$, (a) Cross-sectional area $A$ of bubble plume normalized by $V_{0}$; (b) Air volume $V$ normalized by $V_{0}$; (c) Mean void fraction $\bar{\alpha}_{b}$. Case: $f_{c}=0.88$ $\mathbf{H z}, a k_{c}=0.38$, and $\Delta f / f_{c}=0.73$. Solid curves are functional fits to laboratory data from LM91. Model results shown as open circles.

liquid velocity and oscillation period of the bubble plume measured in the laboratory experiment. Ma et al. also showed an application of their model to predicting breaking wave-induced bubbly flows measured by Cox and Shin (2003). The model/data comparisons of time series of void fraction at different measurement points in the water column are encouraging. Figure 2 shows an example of such comparisons in which dots represent measured data and solid lines represent numerical results.

The foam layer model has been implemented with a wetting-drying scheme. Figure 3 shows a simple test in which the foam layer model was coupled with the NearCoM model (Shi et al., 2005). Monochromatic waves were applied on an idealized barred beach bathymetry with rip channels. The left panel of Figure 3 shows wave crests predicted by the REF/DIF-1 wave module (Kirby et al., 2005). The right panel shows the foam pattern (color) predicted by the foam layer model and the nearshore circulation predicted by the nearshore circulation model SHORECIRC (Svendsen et al., 2004, Shi et al., 2003). Foam patches are driven by waves and nearshore currents, exhibiting onshore/offshore flow patterns including 

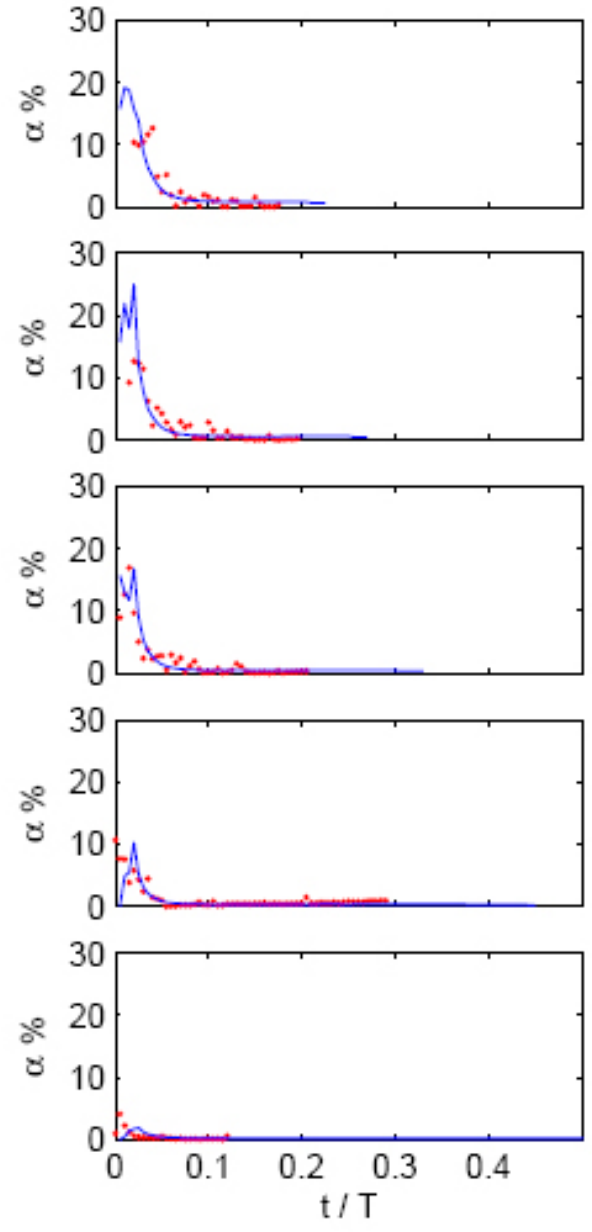

Figure 2. Comparison of simulated and measured void fractions at the measurement array (from top to bottom: $2.5,1.5,0.5,-0.5,-1.5 \mathrm{~cm}$ in respect to the still water level).

the onshore movement driven by waves and offshore transport convected by rip currents. The foam generation and bursting rates were artificially set up in the test and their formulations need to be further investigated.

\section{CONCLUSIONS}

We have developed and modified several numerical models for predicting physical processes associated with optical properties inside the surfzone. The models were tested again laboratory experimental data. The model theories and 

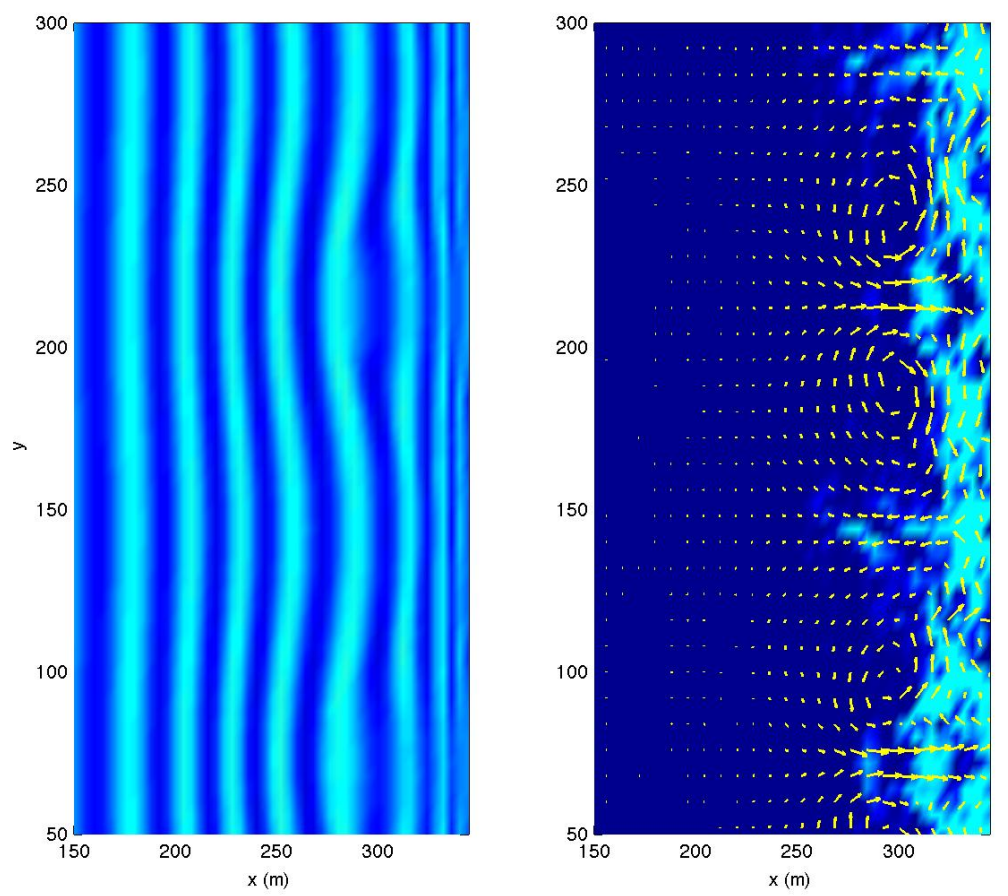

Figure 3. (Left) wave crests; (right) foam pattern and wave-induced nearshore circulation (vectors).

performance are summarized as follows.

1) The multiphase bubble models using the prescribed air entrainment formulation skip directly modeling of the air entrainment process which may be computational unaffordable in a surfzone-scale domain. The models fed with the initially entrained bubbles basically simulates bubble plumes, and requires much less spatial and temporal resolution than needed to capture detailed air entrainment process.

2) The air entrainment formulation developed in Shi et al. (2010) contains a dimensional parameter which limits its applications to general scales. Ma et al. (2010) derived a more theoretical adjustable formulation based on the physical analysis of bubble creation processes.

3) For modeling quiescent phase of air bubbles, a 2DV model (Shi et al., 2008, 2010) and a 3D model (Ma et al., 2010) were developed based on Buscaglia et al (2002) and Carrica et al. (1998), respectively. Ma et al.'s model is a two-fluid model based on the theory of the two-phase flow with taking into account the 
air-liquid momentum transfer. Shi et al.'s model is a two-fluid model based on the continuum mixture theory. Although it is less rigorous in theory compared with Ma et al.'s model, the mixture-type model still remains a valuable alternative as a computational efficient model for practical purposes.

4) Model tests showed that the air entrainment parameter calibrated using experimental data results in generally good agreement between predicted and measured void fractions. Shi et al. (2010) reported an under-prediction of void fraction at the beginning of wave breaking which was not found in Ma et al. (2010). The under-prediction is suspected to be caused by the single-phase model (RIPPLE) used for the mixture phase.

5) The two-fluid models do not account for the entrainment of identifiable gas pockets during the early stages of breaking, and the contribution of these pockets to initial average void fraction is absent. It is necessary to develop an algorithm which moves entrained air volumes from a discrete two-phase representation into the continuum multiphase representation, in order to continue computations without requiring the VOF algorithm to maintain the identity of larger entrained bubbles.

6) We are developing a foam layer model which describes the surface foam dynamics. The source/sink terms in the governing equations are believed to be crucial due to the lack of sufficient knowledge of foam generation and bursting. The formulations for the source/sink terms need to be developed using parameterized results from the two-fluid model or field data if available.

\section{ACKNOWLEDGMENTS}

This study was supported by Office of Naval Research, Coastal Geosciences Program, grants N00014-07-1-0582, N00014-09-1-0853, and N00014-10-1-0088.

\section{REFERENCES}

Buscaglia, G. C., Bombardelli, F. A., and Garcia, M H., 2002, Numerical modeling of large-scale bubble plumes accounting for mass transfer effects, International Journal of Multiphase Flow, 28, 1763-1785

Carrica, P. M., Bonetto, F., Drew, D. A., Lahey, R. T. Jr, 1998, The interaction of background ocean air bubbles with a surface ship, Int. J. Numer. Meth. Fluids, 28, 571-600.

Chen, P., Sanyal, J., and Duduković, M. P., 2005, Numerical simulation of bubble columns flows: effect of different breakup and coalescence closures, Chemical Engineering Science, 60, 1085-1101.

Clift, R., Grace, J., Weber, M., 1978, Bubbles, Drops and Particles, Academic Press.

Cox, D. and Shin, S., 2003, Laboratory measurements of void fraction and turbulence in the bore region of surf zone waves, J. Eng. Mech., 129, 1197-1205

Davini C., 2010, A continuum model for fluid foams, Journal of Elasticity, 101, 77-99. 
Deen N.G., Solberg T. and Hjertager B.H., 2001, Large eddy simulation of the gas-liquid flow in a square cross-sectioned bubble column, Chem. Engng Sci., 56, 6341-6349

Deane G.B. and Stokes M.D., 1999, Air entrainment processes and bubble size distributions in the surf zone, J. Phys. Oceanogr., 29, 1393-1403

Deane, G. B. and Stokes, M. D., 2002, Scale dependence of bubble creation mechanisms in breaking waves, Nature 418, 839-844.

Drew, D and Passman, S., 1998, Theory of multicomponent fluids, Springer.

Francois, M. M., Cummins, S. J., Dendy, E. D., Kothe, D. B., Sicilian, J. M., and Williams, M. W., 2006, A balanced-force algorithm for continuous and sharp interfacial surface tension models within a volume tracking framework, Journal of Computational Physics, 213, 141-173.

Garrett, C., Li M., and Farmer, D., 2000, The connection between bubble size spectra and energy dissipation rates in the upper ocean, Journal of Physical Oceanography, 30, 2163-2171.

Hinze, J. O., 1955, Fundamentals of the hydrodynamic mechanism of splitting in dispersion processes, Amer. Inst. Chem. Eng. J., 1, 289-295.

Hoque, A, 2002. Air bubble entrainment by breaking waves and associated energy dissipation, PhD Thesis, Tokohashi University of Technology.

Kataoka, I. and Serizawa, A., 1989, Basic equations of turbulence in gas-liquid two-phase flow, Int. J. Multiphase Flow 15, pp. 843-855.

Kirby, J. T., Dalrymple, R. A. and Shi, F., 2005, Combined refraction/diffraction model REF/DIF 1, Version 3.0. Documentation and user's manual, Research Report, Center for Applied Coastal Research, University of Delaware.

Kothe, D. B., Mjolsness, R. C. and Torrey, M. D., 1991, RIPPLE: a computer program for incompressible flows with free surfaces, Los Alamos National Laboratory, Report LA - 12007 - MS.

Lamarre, E. and Melville, W. K., 1991, Air entrainment and dissipation in breaking waves, Nature, 351, 469-472.

Lamarre, E. and Melville, W. K., 1994, Void-fraction measurements and sound-speed fields in bubble plumes generated by breaking waves, $J$. Acoust. Soc. Am., 95 (3), 1317-1328.

Lasheras, J. C., Eastwood, C., Martínez-Bazán, and Montanñes, J. L., 2002, A review of statistical models for the break-up of an immiscible fluid immersed into a fully developed turbulent flow, International Journal of Multiphase Flow, 28, 247-278.

Lin, P. and Liu, P. L.-F, 1998, A numerical study of breaking waves in the surf zone, J. Fluid Mech., 359, pp. 239-264.

Liu, D. and Lin, P., 2008, A numerical study of three-dimensional liquid sloshing in tanks, Journal of Computational Physics, 227, 3921 - 3939. 
Luo, H. and Svendsen, H. F., 1996, Theoretical model for drop and bubble breakup in turbulent dispersions, AIChE J., 42, 1225-1233.

Ma, G., Shi, F., and Kirby, J. T., 2010, A polydisperse two-fluid model for bubble plume under breaking waves, Proc. $7^{\text {th }}$ International Conference on Multiphase Flow, Tampa, FL, May 30 - June 4.

Martínez-Bazán, C., Montañes, J.L., Lasheras, J.C., 1999, On the break-up of an air bubble injected into a fully developed turbulent flow. Part I: Break-up frequency, J. Fluid Mech. 401, 157-182.

Moraga, F. F., Carrica, P. M., Drew, D. A., and Lahey Jr., R. T., 2008, A sub-grid air entrainment model for breaking bow waves and naval surface ships, Computers and Fluids, 37, 281-298.

Mori, N., Suzuki, T. and Kakuno, S., 2007, Experimental study of air bubbles and turbulence characteristics in the surf zone, J. Geophy. Res., 112, C05014, doi:10.1029/2006JC003647.

Prince, M.J. and Blanch, H.W., 1990, Bubble coalescence and break-up in air-sparged bubble columns, AICHE J., 36, 1485-1499

Sato Y. and Sekoguchi K., 1975, Liquid velocity distribution in two-phase bubble flow, Int. J. Multiphase Flow, 2, 79-95.

Shi, F., Svendsen, I. A., Kirby, J. T., and Smith, J. M., 2003, A curvilinear version of a quasi-3D nearshore circulation model, Coastal Engineering, $49,99-124$

Shi, F., Kirby, J. T., Newberger, T. A., and Haas, K., 2005, NearCoM master program, version 2005.4, User's manual and module integration, Research Report 2005-10, Center for Appl. Coastal Res., Univ. of Del., Newark.

Shi, F., Kirby, J. T., Haller, M. C. and Catalan, P., 2008, Modeling of surfzone bubbles using a multiphase VOF model, Proc. 31st Int. Conf. Coastal Engrng., Hamburg, 157-169.

Shi, F., Kirby, J. T., and Ma, G., 2010, Modeling quisecent phase transport of air bubbles induced by breaking waves, Ocean Modelling, 35, 105-117.

Smagorinsky J., 1963, General circulation experiments with the primitive equations, Mon. Weather Rev., 91, 99-165.

Svendsen, I. A., Haas, K., and Zhao, Q., 2004, Quasi-3D Nearshore Circulation Model SHORECIRC: Version 2.0, Research Report, Center for Applied Coastal Research, University of Delaware.

Terrill, E. J., Melville, W. K., and Stramski, D., 2001, Bubble entrainment by breaking waves and their influence on optical scattering in the upper ocean, J. Geophys. Res., 106, C8, 16,815-16,823.

Thorpe, S. A., 1982, On the clouds of bubbles formed by breaking wind-waves in deep water, and their role in air-sea gas transfer, Phil. Trans. Roy. Soc. London A, 304, 155-210.

Troshko A.A. and Hassan Y.A., 2001, A two-equation turbulence model of turbulent bubbly flows, Int. J. Multiphase Flow, 27, 1965-2000

Weaire, D. and Hutzler, S., 1999, The physics of foam, Clarendon, Press, Oxford. 